\title{
Characterization of fibroblast growth factor 1 in obese children and adolescents
}

\author{
Anru Wang 1,2, Xueqin Yan', Cai Zhang', Caiqi Du', Wenjun Long', Di Zhan' and Xiaoping Luo' \\ 'Department of Pediatrics, Tongji Hospital of Tongji Medical College, Huazhong University of Science and Technology, Wuhan, China \\ ${ }^{2}$ Department of Pediatrics, The Second Affiliated Hospital of Nanjing Medical University, Nanjing, China \\ ${ }^{3}$ Department of Pediatrics, Boai Hospital of Zhongshan, Zhongshan, China \\ Correspondence should be addressed to X Luo: xpluo@tjh.tjmu.edu.cn
}

\begin{abstract}
Background: Fibroblast growth factor 1 (FGF1) can regulate glucose and lipid metabolism in obese mice. Serum FGF1 has increased in type 2 diabetes mellitus adults and correlated with BMI. This study aimed to indicate conventional weight loss effects on FGF1 in obese children and adolescents.

Materials and methods: Clinical and metabolic parameters of 88 lean and obese individuals (ages 5-15 years) and 39 obese individuals followed with 6 months of lifestyle intervention were collected. Serum FGF1 levels were detected through enzyme-linked immunosorbent assays.

Results: FGF1 levels were increased in obese individuals. Serum FGF1 levels were significantly correlated with $\mathrm{BMI}$ and waist circumferences $(r=0.377, P=0.012 ; r=0.301$, $P=0.047$, respectively). Multivariate stepwise linear regression analyses showed that FGF1 levels were significantly correlated with HbA1c and HOMA-IR ( $\beta=0.371, P=0.008$; $\beta=0.323, P=0.021$, respectively). Weight loss $(2.3 \pm 0.1 \mathrm{~kg})$ was accompanied by a significant reduction of circulating FGF1 levels $(7.2 \pm 0.4 \mathrm{pg} / \mathrm{mL})$. Changes in FGF1 were significantly correlated with changes in fasting glucose, HOMA-IR and low-density lipoprotein cholesterol $(\beta=0.277, P=0.020 ; \beta=0.474, P<0.001 ; \beta=0.320, P=0.008$, respectively).

Conclusion: FGF1 was related to increased risk of insulin resistance in obese children and adolescents. Serum FGF1 reduced after weight loss in obese individuals and was associated with the improvement of insulin resistance. Changes in serum FGF1 were more correlated with insulin resistance than changes in obesity per se.
\end{abstract}

\author{
Key Words \\ - fibroblast growth factor 1 \\ - weight loss \\ - children and adolescents \\ - obesity
}

Endocrine Connections (2018) 7, 932-940

\section{Introduction}

The worldwide prevalence of obesity among children and adolescents has increased in past years (1). Obesity is associated with insulin resistance, type 2 diabetes mellitus (T2DM) and long-term cardiovascular complications. Recent clinical and animal studies have reported that FGF19 and FGF21 are associated with obesity and T2DM $(2,3)$. FGF19 and FGF21 suppress triglyceride and glucose syntheses, meanwhile inducing glycogenesis and fatty acid oxidation in the liver. Recently, FGF1, a mitogenic factor, has been shown to modulate energy homeostasis

$$
\begin{array}{lr}
\text { http://www.endocrineconnections.org } & \text { ( ) } 2018 \text { The authors } \\
\text { https://doi.org/10.1530/EC-18-0141 } & \text { Published by Bioscientifica Ltd }
\end{array}
$$

(4). FGF1 decreases blood glucose levels and improves insulin sensitivity in animals $(5,6)$. Exogenous FGF1 can improve metabolic disorders in rodents through endocrine manner (5). In adults, circulating FGF1 levels are reported to be significantly reduced in obese individuals (7), but significantly increased in T2DM patients (8). Serum FGF1 levels were positively correlated with BMI, waist circumference (WC), fasting glucose and hemoglobin A1c (HbA1c) in T2DM adults (8). The correlation between FGF1 and other glucose/lipid metabolic variables following

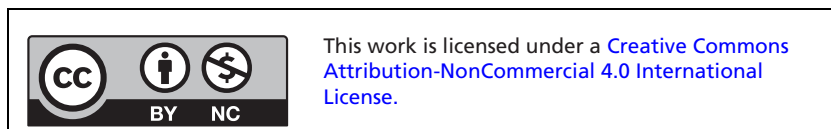


weight loss in obese individuals remains unknown. Therefore, in this study, we aimed to investigate the putative role of FGF1 in the obese youth, assess the influence of lifestyle-induced weight loss on serum FGF1 levels and the relationships between FGF1 and other metabolic factors.

\section{Subjects and methods}

\section{Subjects and baseline data}

Forty-four obese children and adolescents (5-15 years of age) were recruited from Boai Hospital of Zhongshan, and 44 age- and sex-matched normal-weight healthy children and adolescents from the child health care department were also enrolled as controls. In total, there were 45 boys and 43 girls. Obesity was defined as age- and sexspecific BMI of greater than or equal to 95th percentile in accordance with the growth charts for children in China (9). BMI values were converted to standard deviation score (SDS) values in children and adolescents using the lambda-mu-sigma method (9). Insulin resistance was evaluated using the homeostatic model assessment of insulin resistance (HOMA-IR) index (10). Insulin resistance was defined as greater than or equal to the age- and sexspecific 75th percentile HOMA-IR in accordance with the China Health and Nutrition Surveys (CHNS), as described previously (11). Obese individuals were further classified as follows: the insulin-resistant (IR) group $(n=18)$ and the non-IR group $(n=26)$.

Obese children and adolescents whose weight change more than 5\% after 6 months of lifestyle intervention were included in subsequent studies. Those individuals treated with medications or weight loss less than 5\% were excluded. Five obese children and adolescents were excluded from the following study. These individuals included four patients treated with metformin and one child with less than 5\% weight loss. Lifestyle intervention includes moderate caloric restriction and daily physical activity, refer to clinical practice guidelines $(12,13)$. Individuals who had specific medical diagnoses (e.g., hypothyroidism, Cushing's syndrome or polycystic ovary syndrome) and/or current use of medications that may affect body composition or lipid and glucose metabolism (e.g., the use of thyroid medication, thiazolidinediones or metformin) were excluded.

All anthropometric and biochemical measures were recorded at baseline and after 6 months of the intervention. Fasting serum samples previously preserved in outpatient visits were collected to determined FGF1 levels. Serum

$$
\text { http://www.endocrineconnections.org }
$$

FGF1 levels were determined through a sandwich enzymelinked immunosorbent assay (ELISA; Cloud-Clone Corp., Wuhan, China). Detection range is from 15.6 to $1000 \mathrm{pg} / \mathrm{mL}$. The standard curve concentrations used for the ELISA were $1000,500,250,125,62.5,31.2$ and $15.6 \mathrm{pg} / \mathrm{mL}$. The minimum detectable dose of FGF1 is typically less than $6.5 \mathrm{pg} / \mathrm{mL}$. All samples were analyzed in triplicate, and samples with a coefficient of variation of more than $15 \%$ were excluded.

Written informed consent was obtained from the parents or other guardians in accordance with the tenets of the Declaration of Helsinki. The Ethics Committee of Boai Hospital of Zhongshan approved the study protocol.

\section{Statistical analysis}

All data are shown as the means \pm standard errors of the means (S.E.M.S). SPSS version 18.0 for Windows (SPSS Inc) was used for statistical analysis of the data. Comparisons of continuous variables between two groups were carried out using independent sample t-tests. Paired samples $t$-tests were used to compare clinical data before and after weight loss. Categorical variables were analyzed using the chi-square test. The variables were tested for normality with the Shapiro-Wilk test. Non-normally distributed parameters were logarithmically transformed before correlation analysis in the obese group. Pearson correlations tests were performed for bivariate correlation analyses. Multivariate stepwise linear regression analysis was conducted to explore the association of FGF1 with other metabolic variables. The multivariate regression model included variables significantly associated with FGF1 at the bivariate correlation analyses. Two-tailed $P$ values of less than 0.05 were considered significant.

\section{Results}

\section{Baseline characterization of all individuals}

The anthropometric and biochemical characteristics of all the participants are shown in Table 1 . There were no significant differences in age, sex or height between the obese and control groups. Obese children and adolescents had higher levels of obesity descriptors, such as BMI, BMI $Z$-score and WC. Serum low-density lipoprotein cholesterol (LDL-C) levels were higher in obese individual than in normal-weight controls $(2.26 \pm 0.04$ vs $2.48 \pm 0.07$, $P=0.008)$. Fasting insulin and HOMA-IR were higher in the obese group than in healthy controls.

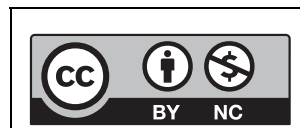

This work is licensed under a Creative Commons Attribution-NonCommercial 4.0 International License. 
Table 1 Baseline data and serum FGF1 levels for the obese children and adolescents and the normal-weight healthy controls.

\begin{tabular}{l} 
Variable \\
\hline Age (years) \\
Sex (boy:girl) \\
Height (cm) \\
Weight (kg) \\
BMI (kg/m²) \\
BMI z-score \\
WC (cm) \\
FG (mmol/L) \\
Fasting insulin (mIU/L) \\
HOMA-IR \\
HbA1c (\%) \\
TC (mmol/L) \\
TG (mmol/L) \\
HDL-C (mmol/L) \\
LDL-C (mmol/L) \\
Apo-A (g/L) \\
Apo-B (g/L) \\
ALT (IU/L) \\
FGF1 (pg/mL) \\
NAFLD
\end{tabular}

\begin{tabular}{c}
\hline \\
\hline Controls $(n=44)$ \\
\hline $9.45 \pm 0.33$ \\
$24: 20$ \\
$134.9 \pm 1.8$ \\
$30.8 \pm 1.1$ \\
$16.6 \pm 0.2$ \\
$0.10 \pm 0.04$ \\
$60.0 \pm 1.2$ \\
$4.78 \pm 0.08$ \\
$6.96 \pm 0.29$ \\
$1.49 \pm 0.07$ \\
$4.99 \pm 0.06$ \\
$4.19 \pm 0.09$ \\
$1.08 \pm 0.05$ \\
$1.56 \pm 0.05$ \\
$2.26 \pm 0.04$ \\
$1.27 \pm 0.04$ \\
$0.80 \pm 0.02$ \\
$16.9 \pm 0.8$ \\
$12.7 \pm 0.2$ \\
0
\end{tabular}

Total

Obesity $(n=44)$

$9.51 \pm 0.34$

$21: 23$

$139.7 \pm 1.9$

$46.0 \pm 2.0$

$23.1 \pm 0.4$

$2.42 \pm 0.08$

$77.7 \pm 1.9$

$4.99 \pm 0.06$

$13.64 \pm 1.33^{a}$

$3.03 \pm 0.30^{\mathrm{a}}$

$5.29 \pm 0.04$

$4.27 \pm 0.10$

$0.97 \pm 0.05$

$1.50 \pm 0.05$

$2.48 \pm 0.07$

$1.21 \pm 0.03$

$0.78 \pm 0.02$

$22.8 \pm 2.0$

$30.0 \pm 1.0$

$13(30 \%)$

\begin{tabular}{c} 
\\
\hline Non-IR $(n=26)$ \\
\hline $9.83 \pm 0.50$ \\
$13: 13$ \\
$140.9 \pm 3.0$ \\
$47.7 \pm 3.0$ \\
$23.4 \pm 0.5$ \\
$2.37 \pm 0.09$ \\
$77.9 \pm 2.7$ \\
$4.92 \pm 0.09$ \\
$8.68 \pm 0.64^{\mathrm{a}}$ \\
$1.89 \pm 0.14$ \\
$5.29 \pm 0.05$ \\
$4.36 \pm 0.14$ \\
$0.90 \pm 0.06$ \\
$1.57 \pm 0.06$ \\
$2.50 \pm 0.10$ \\
$1.27 \pm 0.05$ \\
$0.79 \pm 0.03$ \\
$18.1 \pm 1.5$ \\
$27.7 \pm 1.0$ \\
$1(0.04 \%)$ \\
\end{tabular}

Obesity

\begin{tabular}{c}
$P$ \\
\hline 0.906 \\
$N S$ \\
0.066 \\
0.000 \\
0.000 \\
0.000 \\
0.000 \\
0.510 \\
0.000 \\
0.000 \\
0.000 \\
0.548 \\
0.101 \\
0.361 \\
0.008 \\
0.278 \\
0.375 \\
0.000 \\
0.000
\end{tabular}

IR

$9.05 \pm 0.38$

$8: 10$

$138.1 \pm 1.9$

$43.6 \pm 2.0$

$22.7 \pm 0.5$

$2.47 \pm 0.13$

$77.4 \pm 2.6$

$5.08 \pm 0.08$

$20.80 \pm 2.23^{\mathrm{a}}$

$4.67 \pm 0.50^{\mathrm{a}}$

$5.29 \pm 0.06$

$4.14 \pm 0.16$

$1.07 \pm 0.07$

$1.40 \pm 0.06$

$2.46 \pm 0.10$

$1.12 \pm 0.04$

$0.75 \pm 0.03$

$29.4 \pm 4.0$

$33.1 \pm 1.8$

$12(66.6 \%)$

Baseline data are given as means \pm S.E.M.S.

${ }^{a}$ Data not normally distributed; ${ }^{\text {b }}$ chi-square analysis.

ALT, alanine aminotransferase; Apo-A, apolipoprotein A; Apo-B, apolipoprotein B; FG, fasting glucose; FGF1, fibroblast growth factor 1; HbA1C, hemoglobin A1C; HDL-C, high-density lipoprotein cholesterol; HOMA-IR, homeostasis model assessment of insulin resistance; IR, insulin-resistant; LDL-C, low-density lipoprotein cholesterol; NAFLD, non-alcoholic fatty liver disease; NS, not significant; TC, total cholesterol; TG, triglyceride; WC, waist circumferences.

No significant differences in anthropometric and HbA1c levels were observed between the IR and non-IR obese groups. Apolipoprotein A (Apo-A) was the only lipid metabolism indicator that displayed a difference between the IR and the non-IR obese groups $(1.27 \pm 0.05$ vs $1.12 \pm 0.04, P=0.031)$. There were no sex-specific differences in age, BMI $z$-score and WC (data not shown).

\section{Features of FGF1 in different groups}

A significant increase of serum FGF1 levels was observed in obese children and adolescents compared to that in the control group (Fig. 1A and Table 1). Circulating FGF1 levels were higher in the IR obese group than in the non-IR obese group (Fig. 1B). To assess the influence of obesity on FGF1, we analyzed the correlation between serum FGF1 levels and other variables in the obese group. FGF1 levels were significantly positively correlated with anthropometric parameters in the obese group, such as BMI, BMI $z$-score and WC $(r=0.377$, $P=0.012 ; \quad r=0.407, \quad P=0.006 ; \quad r=0.301, \quad P=0.047$, respectively; Fig. 1C and D). Circulating FGF1 levels were significantly correlated with LDL-C in the obese group ( $r=0.367, P=0.014$, Fig. 1E). Fasting insulin and HOMA-IR that were not normally distributed in the obese group were logarithmically transformed to normally distributed data before correlation analysis. Serum FGF1 levels were significantly correlated with glucose metabolic factors, including $\mathrm{HbA1c}$, fasting insulin and HOMA-IR in the obese group $(r=0.411$, $P=0.006 ; \quad r=0.396, \quad P=0.008 ; \quad r=0.386, \quad P=0.010$, respectively; Fig. $1 \mathrm{~F}, \mathrm{G}$ and $\mathrm{H}$ ). To characterize the relationship between FGF1 and obesity further, a multiple stepwise regression analysis was performed using FGF1 as dependent variables, whereas BMI, WC, HbA1c, HOMA-IR and LDL-C were used as independent variables. As shown in Table 2, HbA1c and HOMA-IR were strongly related to serum FGF1 levels.

Grouping by sex, serum FGF1 levels were increased in the obese boys $(30.0 \pm 1.4$ vs $12.9 \pm 0.3 \mathrm{pg} / \mathrm{mL})$ and girls $(29.9 \pm 1.5$ vs $12.4 \pm 0.3 \mathrm{pg} / \mathrm{mL})$. Serum FGF1 levels were significantly correlated with BMI and BMI $z$-score in boys $(r=0.825, P<0.001 ; r=0.850, P<0.001$, respectively) and girls $(r=0.897, P<0.001 ; r=0.920$, $P<0.001$, respectively). In boys, serum FGF1 levels were correlated with HOMA-IR after adjusting for age and BMI $(r=0.414, P<0.05)$.

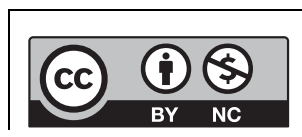

This work is licensed under a Creative Commons Attribution-NonCommercial 4.0 International License. 

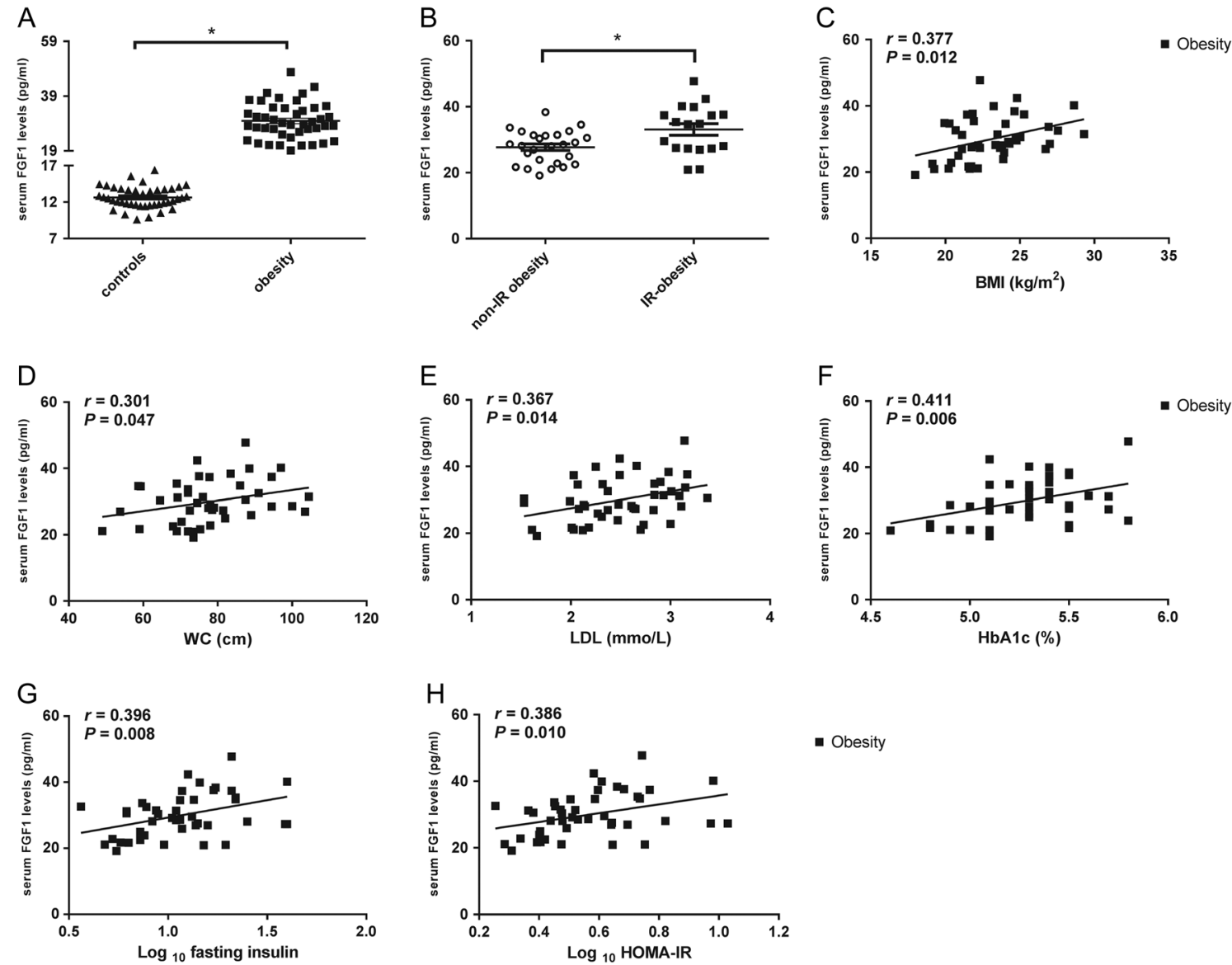

\section{Figure 1}

Comparison of circulation fibroblast growth factor 1 (FGF1) in different conditions. (A) Circulating FGF1 levels comparison between the obese children and adolescents ( $n=44)$ and the normal-weight healthy controls $(n=44)$; (B) serum FGF1 levels comparison between the insulin-resistant (IR) $(n=18)$ and non-IR obese $(n=26)$ groups; (C) serum FGF1 levels were significantly correlated with BMI in the obese group; (D) circulating FGF1 levels were significantly correlated with waist circumference (WC) in the obese group; (E) serum FGF1 levels were significantly correlated with low-density lipoprotein cholesterol (LDL-C) in the obese group; (F) circulating FGF1 levels were significantly correlated with hemoglobin A1c (HbA1c) in the obese group; (G) FGF1 levels were significantly correlated with logarithmically transformed fasting insulin in obese individuals; (H) serum FGF1 levels were significantly correlated with logarithmic transformed homeostatic model assessment of insulin resistance (HOMA-IR) in the obese group. Data are shown as mean \pm S.E.M. ${ }^{*} P<0.05$.

\section{Effects of weight loss in obese individuals}

Clinical and laboratory parameters at baseline and after intervention were determined in 39 obese individuals (Table 3). The 6 month of intervention reduced body weight by $2.3 \pm 0.1 \mathrm{~kg}(45.2 \pm 2.1$ vs $42.8 \pm 2.0 ; P<0.001)$. Other anthropometric parameters, such as BMI and BMI $z$-score were significantly decreased after 6 months. The changes in weight during 6 months were associated with an improvement in glucose and lipid metabolism. Glucose metabolic variables, including fasting insulin, HOMA-IR and $\mathrm{HbA} 1 \mathrm{c}$, were apparently decreased. TC and Apo-B, as lipid metabolic indexes, were significantly decreased after treatments. Other metabolic variables, such as fasting glucose (FG), TG, LDL-C and Apo-A, were decreased, while HDL-C was increased after the intervention. Interestingly,

$$
\begin{array}{lr}
\text { http://www.endocrineconnections.org } & \text { () } 2018 \text { The authors } \\
\text { https://doi.org/10.1530/EC-18-0141 } & \text { Published by Bioscientifica Ltd }
\end{array}
$$

serum FGF1 levels were significantly decreased by $7.2 \pm 0.4 \mathrm{pg} / \mathrm{mL}(29.4 \pm 1.0$ vs $22.2 \pm 0.7 ; P<0.001)$ after weight loss. We next performed Pearson analyses for bivariate correlations between changes in FGF1 and changes in other metabolic indexes. Changes in FGF1 levels from baseline to 6 months were directly correlated with changes in BMI $(r=0.427, P=0.007$; Fig. 2B). The change in FGF1 was positively correlated with the change in weight $(r=0.320, P=0.047)$. Changes in FGF1 levels were also significantly correlated with changes in FG, fasting insulin, HOMA-IR and LDL-C $(r=0.443, P=0.005$; $r=0.672, P<0.001 ; r=0.648, P<0.001 ; r=0.486, P=0.002$, respectively; Fig. 2C, D, E and F). Multiple stepwise linear regression analysis was performed with changes in FGF1 as the dependent variables. Changes in BMI, FG, HOMA-IR and LDL-C were used as independent variables

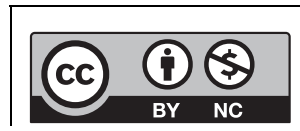

This work is licensed under a Creative Commons Attribution-NonCommercial 4.0 International License. 
Table 2 Multivariate stepwise linear regression analysis for serum FGF1 levels in obese children and adolescents.

\begin{tabular}{|c|c|c|c|}
\hline \multirow{2}{*}{$\begin{array}{l}\text { Independent } \\
\text { variable }\end{array}$} & \multirow[b]{2}{*}{$\beta$} & \multicolumn{2}{|c|}{ FGF1 } \\
\hline & & $95 \% \mathrm{Cl}$ & $P$ \\
\hline HbA1c (\%) & 0.371 & $0.100-0.643$ & 0.008 \\
\hline $\log _{10}$ HOMA-IR & 0.323 & $0.052-0.594$ & 0.021 \\
\hline$R^{2}$ & 0.272 & & \\
\hline
\end{tabular}

Dependent variable: FGF1; independent variables: BMI, WC, HbA1C, $\log _{10}$ HOMA-IR, LDL-C; excluded variables: BMI, WC, LDL-C.

FGF1, fibroblast growth factor 1; HbA1C, hemoglobin A1C; LDL-C, low-density lipoprotein cholesterol; $\log _{10}$ HOMA-IR, logarithmic transformation for homeostasis model assessment of insulin resistance; WC, waist circumferences.

in the regression model. A significant association between changes in FGF1 and changes in the three metabolic indexes was found (Table 4).

\section{Discussion}

In the present study, we analyzed serum FGF1 levels in obese children and adolescents and compared them with those in normal-weight healthy controls. We found that the serum FGF1 levels were strongly elevated in obese individuals than in healthy controls. Furthermore, circulating FGF1 levels were significantly higher in IR obese children and adolescents than non-IR obese individuals. Remarkably, baseline FGF1 levels were significantly correlated with HbA1c and HOMA-IR. We next analyzed the relationship between serum FGF1 levels and other metabolic parameters following weight loss in obese children and adolescents. Weight loss was accompanied by a significant reduction of circulating FGF1 levels. Changes in FGF1 were strongly correlated with changes in FG, HOMA-IR and LDL-C after 6 months of treatments. To the best of our knowledge, the present study is the first to analyze FGF1 levels and their relationship with metabolic indexes in obese children and adolescents. Furthermore, there were few studies evaluating the changes of FGF1 during conventional weight loss.

The mammalian FGF family comprises 22 members and mediates a range of biological responses including development, wound healing, homeostasis and metabolism (14). Most FGFs, called canonical FGFs, interact with heparin or heparin sulfate glycosaminoglycans (HSGAGs) and then activate FGF receptors (FGFR1-4) (15). Roles of FGF19 and FGF21 in glucose and lipid metabolism have been well established $(16,17,18,19)$. Owing to low HSGAGs affinity, both FGF19 and FGF21 require co-receptors such as $\beta$-klotho (KLB) to bind their cognate FGFRs and ultimately participate in inter-organ endocrine signaling axes (20).

Table 3 Baseline data and serum FGF1 levels for the obese children and adolescents after 6 months of lifestyle intervention ( $n=39$, boys: girls $=18: 21)$.

\begin{tabular}{l} 
Variables \\
\hline Age (years) \\
Height $(\mathrm{cm})$ \\
Weight $(\mathrm{kg})$ \\
BMI $\left(\mathrm{kg} / \mathrm{m}^{2}\right)$ \\
BMI $z-\mathrm{score}$ \\
WC (cm) \\
FG (mmol/L) \\
Fasting insulin (mIU/L) \\
HOMA-IR \\
HbA1c (\%) \\
TC (mmol/L) \\
TG (mmol/L) \\
HDL-C (mmol/L) \\
LDL-C (mmol/L) \\
Apo-A (g/L) \\
Apo-B (g/L) \\
ALT (IU/L) \\
FGF1 (pg/mL)
\end{tabular}

\begin{tabular}{c} 
Before \\
\hline $9.41 \pm 0.37$ \\
$139.0 \pm 2.1$ \\
$45.2 \pm 2.1^{a}$ \\
$22.9 \pm 0.4$ \\
$2.41 \pm 0.08$ \\
$76.3 \pm 1.9$ \\
$4.97 \pm 0.07$ \\
$11.39 \pm 0.85^{a}$ \\
$2.51 \pm 0.19^{a}$ \\
$5.27 \pm 0.04$ \\
$4.29 \pm 0.12$ \\
$0.98 \pm 0.05$ \\
$1.52 \pm 0.05$ \\
$2.47 \pm 0.08$ \\
$1.22 \pm 0.04$ \\
$0.78 \pm 0.02$ \\
$22.5 \pm 2.2^{a}$ \\
$29.4 \pm 1.0$
\end{tabular}

\begin{tabular}{c} 
After \\
\hline $9.91 \pm 0.37$ \\
$140.9 \pm 2.1$ \\
$42.8 \pm 2.0^{a}$ \\
$21.1 \pm 0.4$ \\
$1.73 \pm 0.07$ \\
$75.0 \pm 1.9$ \\
$4.89 \pm 0.07$ \\
$8.66 \pm 0.68^{a}$ \\
$1.89 \pm 0.15^{a}$ \\
$4.97 \pm 0.03$ \\
$3.72 \pm 0.08$ \\
$0.89 \pm 0.04$ \\
$1.55 \pm 0.05$ \\
$2.37 \pm 0.07$ \\
$1.16 \pm 0.04$ \\
$0.69 \pm 0.02$ \\
$19.7 \pm 1.7^{a}$ \\
$22.2 \pm 0.7$
\end{tabular}

\begin{tabular}{c}
\hline Changes \\
\hline 0.5 \\
$2.0 \pm 0.1$ \\
$2.3 \pm 0.1^{\mathrm{a}}$ \\
$1.8 \pm 0.1$ \\
$0.68 \pm 0.02$ \\
$1.3 \pm 0.1$ \\
$0.07 \pm 0.01$ \\
$2.73 \pm 0.28$ \\
$0.63 \pm 0.06$ \\
$0.30 \pm 0.02^{\mathrm{a}}$ \\
$0.57 \pm 0.05$ \\
$0.09 \pm 0.01$ \\
$0.02 \pm 0.01$ \\
$0.10 \pm 0.01$ \\
$0.06 \pm 0.01$ \\
$0.09 \pm 0.01$ \\
$2.9 \pm 0.5^{\mathrm{a}}$ \\
$7.2 \pm 0.4$
\end{tabular}

\begin{tabular}{c}
\multicolumn{1}{c}{$\boldsymbol{P}^{\mathbf{b}}$} \\
\hline \\
$<0.001$ \\
$<0.001$ \\
$<0.001$ \\
$<0.001$ \\
$<0.001$ \\
$<0.001$ \\
$<0.001$ \\
$<0.001$ \\
$<0.001$ \\
$<0.001$ \\
$<0.001$ \\
$<0.001$ \\
$<0.001$ \\
$<0.001$ \\
$<0.001$ \\
$<0.001$ \\
$<0.001$
\end{tabular}

Baseline data are given as means \pm S.E.M.S.

a Data not normally distributed; ${ }^{b}$ paired samples $t$-test. $t$-test for the age variable cannot be computed because the s.E.M. of the difference is zero. ALT, alanine aminotransferase; Apo-A, apolipoprotein A; Apo-B, apolipoprotein B; FG, fasting glucose; FGF1, fibroblast growth factor 1; HbA1C, hemoglobin A1C; HDL-C, high-density lipoprotein cholesterol; HOMA-IR, homeostasis model assessment of insulin resistance; LDL-C, low-density lipoprotein cholesterol; TC, total cholesterol; TG, triglyceride; WC, waist circumferences.

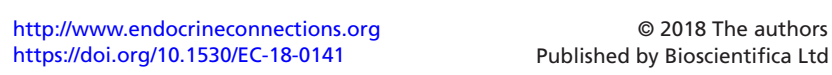


Table 4 Multivariate stepwise linear regression analysis for changes in serum FGF1 levels in obese children and adolescents after 6 months of lifestyle intervention.

\begin{tabular}{|c|c|c|c|}
\hline \multirow{2}{*}{$\begin{array}{l}\text { Independent } \\
\text { variable }\end{array}$} & \multirow[b]{2}{*}{$\beta$} & \multicolumn{2}{|c|}{$\Delta$ FGF1 } \\
\hline & & $95 \% \mathrm{Cl}$ & $P$ \\
\hline$\Delta \mathrm{FG}(\mathrm{mmol} / \mathrm{L})$ & 0.277 & $0.046-0.508$ & 0.020 \\
\hline$\Delta \mathrm{LDL}-\mathrm{C}(\mathrm{mmol} / \mathrm{L})$ & 0.320 & $0.089-0.552$ & 0.008 \\
\hline$\triangle \mathrm{HOMA-IR}$ & 0.474 & $0.233-0.714$ & 0.000 \\
\hline$R^{2}$ & 0.585 & & \\
\hline
\end{tabular}

Dependent variable: changes in serum FGF1 levels; independent variables: changes in BMI, FG, LDL-C and HOMA-IR; excluded variable: changes in BMI.

$\triangle \mathrm{FG}$, changes in fasting glucose; $\triangle \mathrm{FGF} 1$, changes in fibroblast growth factor 1; $\triangle$ HOMA-IR, changes in homeostasis model assessment of insulin resistance; $\Delta \mathrm{LDL}-\mathrm{C}$, changes in low-density lipoprotein cholesterol.

The newly reported metabolic FGF, FGF1, binds tightly to cell HSGAGs and stabilizes the receptor-ligand complex to block FGF1 circulation, thereby leading to autocrine/paracrine signaling $(14,21)$. FGF1 has originally detected expression in rodents and human adipose tissue $(22,23)$. Furthermore, FGF1-knockout mice developed severe hyperglycemia and IR accompanied by elevated inflammation in the adipose tissue when challenged with HFD (22). Interestingly, recent studies suggest that FGF1, as a new therapeutic approach for insulin resistance and T2DM treatment, probably functions in an endocrine manner $(4,5)$. Suh et al. (5) reported that a single subcutaneous injection of recombinant FGF1 (rFGF1) decreased circulating glucose after hours without hypoglycemia in obese mice. Long-term administration of rFGF1 increases glucose uptake in the skeletal muscle, decreases hepatic glucose production, reduces circulating insulin levels, and ultimately improves insulin sensitivity in 3 weeks. Furthermore, a study on adipose tissuespecific Fgfr 1-knockout mice reported that rFGF1 failed to decrease glucose levels, suggesting that adipose tissue is the primary target for exogenous rFGF1. Another study on obese rodents reported that a single central injection of
A
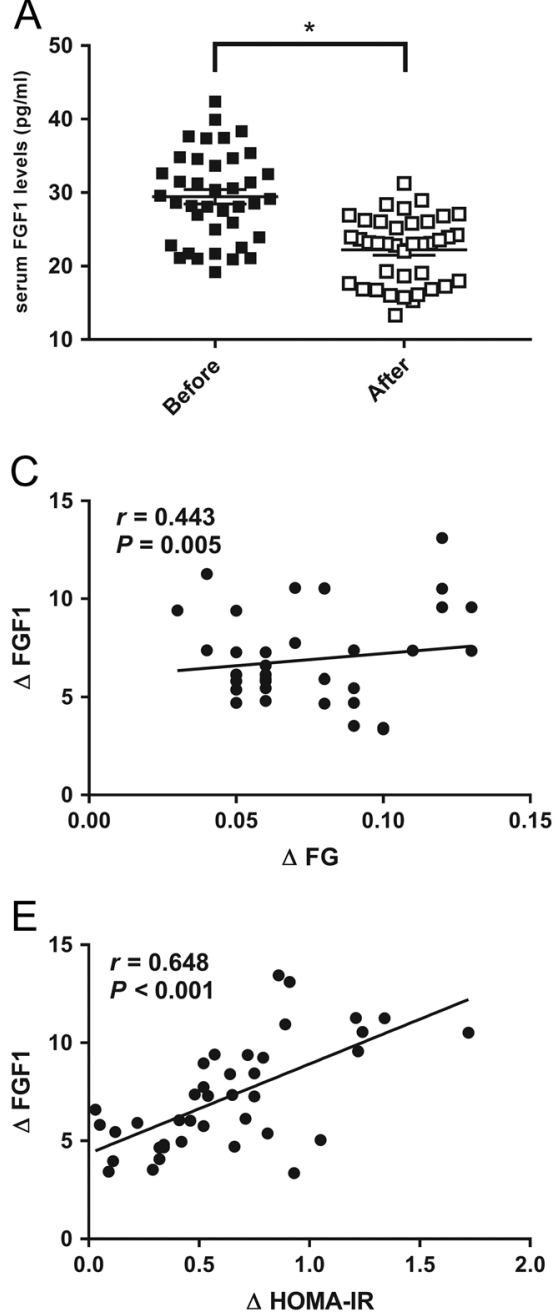

B

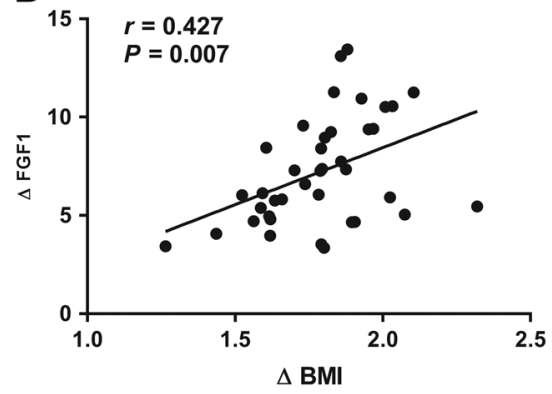

D

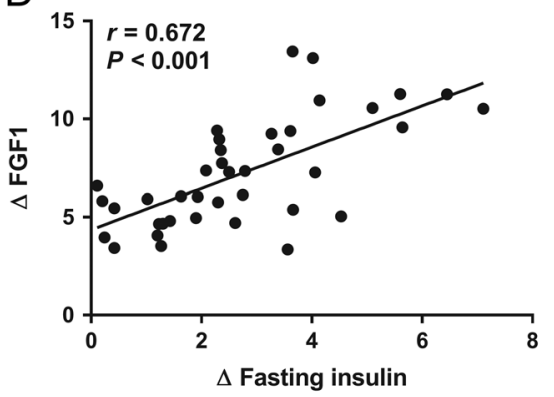

$\mathrm{F}$

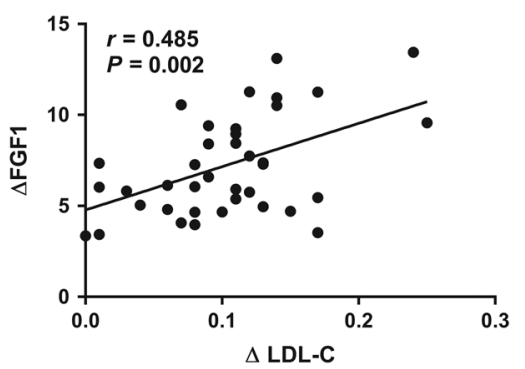

Figure 2

Changes in circulation fibroblast growth factor 1 (FGF1) after 6 months of lifestyle intervention in obese children and adolescents $(n=39)$. (A) Serum FGF1 levels comparison before and after 6 months; (B) changes in serum FGF1 levels were significantly correlated with changes in $\mathrm{BMI}_{\text {; }}$ (C) changes in circulating FGF1 levels were significantly correlated with changes in fasting glucose (FG); (D) changes in serum FGF1 levels were significantly with changes in fasting insulin; (E) changes in FGF1 levels were significantly correlated with changes in homeostatic model assessment of insulin resistance (HOMA-IR); (F) changes in circulating FGF1 levels were significantly correlated with changes in low-density lipoprotein cholesterol (LDL-C). Data are shown as mean \pm S.E.M. ${ }^{*} P<0.05$. http://www.endocrineconnections.org https://doi.org/10.1530/EC-18-0141
() 2018 The authors Published by Bioscientifica Ltd

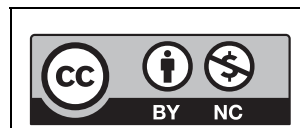

This work is licensed under a Creative Commons Attribution-NonCommercial 4.0 International License. 
rFGF1 induces long-term glucose-lowering effects without changes in insulin sensitivity, probably through different routes of administration compared with peripheral injection (24).

The potential roles of circulating FGF1 in human metabolic disturbances remain unclear. In recent studies, Wang et al. reported that serum FGF1 levels were elevated in adults with newly diagnosed T2DM (8), while Zhu et al. (7) reported that circulating FGF1 levels decreased in obese/overweight adults. Furthermore, serum FGF1 level positively correlated with BMI, WC and waist-to-hip ratio in T2DM adults, but reversely correlated with BMI in obese/overweight subjects $(7,8)$. Our results indicate FGF1 levels were significantly increased in obese children and adolescents compared to normal-weight healthy controls. FGF1 levels in IR obese children and adolescents were elevated compared to the non-IR obese group. Moreover, we found circulating FGF1 to be significantly correlated with BMI. These findings confirmed that increased FGF1 levels may be a risk factor for obese children and adolescents, particularly in individuals with insulin resistance. This view differs from that of Zhu et al. (7) who believe that serum FGF1 levels are a decreased risk of obesity in adults. Notably, there are 20 subjects with type 2 diabetes and ten individuals with anti-diabetes drugs for a total of 71 controls in Zhu's (7) study. FGF1 may play a role in the pathogenesis of type 2 diabetes (8). Comparisons between the obese and lean groups may be biased, in part due to these individuals in the control group. The difference between Zhu's study and our study may be due in part to differences in the grouping methods of the two studies. Furthermore, given that FGF1 may be associated with BMI, different BMI cut-off in overweight and obese children may affect FGF1 levels. In addition, FGF1 levels of all samples in the current study were detected, while Mejhert et al. (25) reported that circulating FGF1 levels in healthy women were not detected. This may be due to the different detection range of the ELISA kits (the lowest standard: $31.3 \mathrm{pg} / \mathrm{mL}$ vs $15.6 \mathrm{pg} / \mathrm{mL}$ ).

It has been reported that fasting glucose and insulin levels were increased in FGF1-/- mice fed with an HFD (22). Wang et al. reported that serum FGF1 significantly correlated with fasting glucose, 2-h post-OGTT glucose and HOMA- $\beta$ (8). In addition, HbA1c were the independent factors influencing FGF1 levels (8). In our study, bivariate correlation analyses indicate that baseline FGF1 levels were positively correlated with BMI, BMI z-score, WC, HbA1c, fasting insulin, HOMA-IR and LDL-C in obese children and adolescents, consistent with Wang's study. Multivariate linear regression analysis indicated that baseline FGF1 levels were strongly correlated with glucose metabolic factors, including HbA1c and HOMA-IR. In addition, baseline FGF1 levels of boys were correlated with HOMA-IR after adjusting for BMI. Thus, the associations between FGF1 and markers of glucose homeostasis were stronger than correlations between FGF1 and anthropometric parameters. Results support the idea that FGF1 plays a role in glucose metabolism in obese children and adolescents, particularly in boys.

We next observed that weight loss after 6 months of lifestyle intervention in obese individuals was companied by improvement of metabolic indexes. Of note, serum FGF1 levels were significantly reduced after weight loss. The weight loss-related reduction of serum FGF1 may be obtained through hypocaloric diet and moderate physical activity. Pearson analyses showed that changes in FGF1 levels were correlated with changes in anthropometric and metabolic parameters, including BMI, FG, fasting insulin, HOMA-IR and LDL-C. Decreases of FGF1 levels may be attributed to lifestyle-induced weight loss and the associated metabolic improvements. Multivariate linear regress analysis indicated that changes in FGF1 were strongly correlated with changes in traits of the metabolic syndrome (FG, HOMA-IR and LDL-C), not with changes in BMI. Serum FGF1 was related to the degree of insulin resistance rather than to obesity (measured by BMI) per se. Weight loss as reported in the present study may decrease circulating FGF1 through an improvement in insulin sensitivity rather than a reduction in BMI.

From the current results, there seems to be some controversy between the benefits of endocrine FGF1 in rodent models and elevated FGF1 concentrations in obese individuals. We supposed that the FGF1 levels in obese children and adolescents may be associated with FGF1 resistance. Further studies are needed to assess the differences between obesity and leanness after rFGF1 administration in rodents. In addition, our study has the following limitations. This was a pilot study with a small number of patients, not a clinical trial. Hence, prospective cohort study with a large sample size is needed to further validate whether therapeutically altering serum FGF1 may represent a useful target for interventions aiming to improve insulin resistance in obese subjects. Considering the limitations of BMI as a measure of obesity, body fat content evaluation by magnetic resonance or dual energy $\mathrm{X}$-ray are needed for further investigation.

Taken together, FGF1 levels were increased in obese children and adolescents compared with lean individuals. Multivariate linear regression analyses show that increased

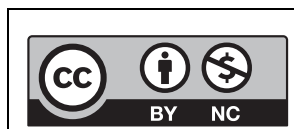

This work is licensed under a Creative Commons Attribution-NonCommercial 4.0 International License. 
serum FGF1 was associated with the development of metabolic syndrome independent of body mass in obese children and adolescents. Six months of lifestyle intervention induced the reduction of serum FGF1. Finally, changes in metabolic indexes were found to be the main and significant predictors of changes in serum FGF1 after weight loss. FGF1 may serve as a potential therapeutic target for insulin resistance in obese children and adolescents.

\section{Declaration of interest}

The authors declare that there is no conflict of interest that could be perceived as prejudicing the impartiality of the research reported.

\section{Funding}

This work was supported by the National Natural Science Foundation of China (grant number 81670781) and program for Changjiang Scholars and Innovative Research Team in University (grant number PCSIRT1131).

\section{References}

1 NCD Risk Factor Collaboration (NCD-RisC). Worldwide trends in body-mass index, underweight, overweight, and obesity from 1975 to 2016: a pooled analysis of 2416 population-based measured studies in 128.9 million children, adolescents, and adults. Lancet 2017390 2627-2642. (https://doi.org/10.1016/S01406736(17)32129-3)

2 Nies VJ, Sancar G, Liu W, van Zutphen T, Struik D, Yu RT, Atkin AR, Evans RM, Jonker JW \& Downes MR. Fibroblast growth factor signaling in metabolic regulation. Frontiers in Endocrinology 20166 193. (https://doi.org/10.3389/fendo.2015.00193)

3 Kharitonenkov A, Shiyanova TL, Koester A, Ford AM, Micanovic R, Galbreath EJ, Sandusky GE, Hammond LJ, Moyers JS, Owens RA, et al. FGF21 as a novel metabolic regulator. Journal of Clinical Investigation 200515 1627-1635. (https://doi.org/10.1172/ JCI23606)

4 Gasser E, Moutos CP, Downes M \& Evans RM. FGF1-a new weapon to control type 2 diabetes mellitus. Nature Reviews Endocrinology 201713 599-609. (https://doi.org/10.1038/nrendo.2017.78)

5 Suh JM, Jonker JW, Ahmadian M, Goetz R, Lackey D, Osborn O, Huang Z, Liu W, Yoshihara E, van Dijk TH, et al. Endocrinization of FGF1 produces a neomorphic and potent insulin sensitizer. Nature 2014513 436-439. (https://doi.org/10.1038/nature13540)

6 Scarlett JM, Rojas JM, Matsen ME, Kaiyala KJ, Stefanovski D, Bergman RN, Nguyen HT, Dorfman MD, Lantier L, Wasserman DH, et al. Central injection of fibroblast growth factor 1 induces sustained remission of diabetic hyperglycemia in rodents. Nature Medicine 2016 22 800-806. (https://doi.org/10.1038/nm.4101)

7 Zhu J, Wang Y, Zhu K, Gao J, Wan X, Pang X \& Fei S. Serum fibroblast growth factor 1 is associated with the decreased risk of obesity in human. Experimental and Clinical Endocrinology and Diabetes 2017125 322-326. (https://doi.org/10.1055/s-0043-104532)

8 Wang S, Yang Q, Yu S, Pan R, Jiang D, Liu Y, Hu H, Sun W, Hong X, Xue $\mathrm{H}$, et al. Fibroblast growth factor 1 levels are elevated in newly diagnosed type 2 diabetes compared to normal glucose tolerance controls. Endocrine Journal 201663 359-365. (https://doi. org/10.1507/endocrj.EJ15-0627)

9 Li H, Ji CY, Zong XN \& Zhang YQ. Body mass index growth curves for Chinese children and adolescents aged 0 to 18 years. Zhonghua
Er Ke Za Zhi 200947 493-498. (https://doi.org/10.3760/cma.j.i ssn.0578-1310.2009.07.004)

10 Brown RJ \& Yanovski JA. Estimation of insulin sensitivity in children: methods, measures and controversies. Pediatric Diabetes 201415 151-161. (https://doi.org/10.1111/pedi.12146)

11 Song P, Yu J, Chang X, Wang M \& An L. Prevalence and correlates of metabolic syndrome in Chinese children: the China health and nutrition survey. Nutrients 20179 E79. (https://doi.org/10.3390/ nu9010079)

12 Subspecialty Group of Endocrinologic, Hereditary and Metabolic Diseases, The Society of Pediatrics, Chinese Medical Association; Subspecialty Group of Cardiology, The Society of Pediatrics, Chinese Medical Association; Subspecialty Groups of Child Health Care, The Society of Pediatrics, Chinese Medical Association. The definition of metabolic syndrome and prophylaxis and treatment proposal in Chinese children and adolescents. Zhonghua Er Ke Za Zhi 201250 420-422. (https://doi.org/10.3760/cma.j.issn.0578-1310.2012. 06.005)

13 Styne DM, Arslanian SA, Connor EL, Farooqi IS, Murad MH, Silverstein JH \& Yanovski JA. Pediatric obesity-assessment, treatment, and prevention: an Endocrine Society Clinical Practice Guideline. Journal of Clinical Endocrinology and Metabolism 2017102 709-757. (https://doi.org/10.1210/jc.2016-2573)

14 Turner N \& Grose R. Fibroblast growth factor signaling: from development to cancer. Nature Review Cancer 201010 116-129. (https://doi.org/10.1038/nrc2780)

15 Degirolamo C, Sabbà C \& Moschetta A. Therapeutic potential of the endocrine fibroblast growth factors FGF19, FGF21 and FGF23. Nature Reviews Drug Discovery 201615 51-69. (https://doi.org/10.1038/ nrd.2015.9)

16 Xie T \& Leung PS. Fibroblast growth factor 21: a regulator of metabolic disease and health span. American Journal of Physiology: Endocrinology and Metabolism 2017313 E292-E302. (https://doi. org/10.1152/ajpendo.00101.2017)

17 Li G, Yin J, Fu J, Li L, Grant SFA, Li C, Li M, Mi J, Li M \& Gao S. FGF21 deficiency is associated with childhood obesity, insulin resistance and hypoadiponectinaemia: the BCAMS Study. Diabetes and Metabolism 201743 253-260. (https://doi.org/10.1016/j. diabet.2016.12.003)

18 Fu L, John LM, Adams SH, Yu XX, Tomlinson E, Renz M, Williams PM, Soriano R, Corpuz R, Moffat B, et al. Fibroblast growth factor 19 increases metabolic rate and reverses dietary and leptindeficient diabetes. Endocrinology 2004145 2594-2603. (https://doi. org/10.1210/en.2003-1671)

19 Hu X, Xiong Q, Xu Y, Zhang X, Pan X, Ma X, Bao Y \& Jia W. Association of serum fibroblast growth factor 19 levels with visceral fat accumulation is independent of glucose tolerance status. Nutrition, Metabolism and Cardiovascular Diseases 201728 119-125. (https://doi.org/10.1016/j.numecd.2017.10.009)

20 Lan T, Morgan DA, Rahmouni K, Sonoda J, Fu X, Burgess SC, Holland WL, Kliewer SA \& Mangelsdorf DJ. FGF19, FGF21, and an FGFR1/ $\beta$-klotho-activating antibody act on the nervous system to regulate body weight and glycemia. Cell Metabolism 201726 709-718. (https://doi.org/10.1016/j.cmet.2017.09.005)

21 Ornitz DM, Xu J, Colvin JS, McEwen DG, MacArthur CA, Coulier F, Gao G \& Goldfarb M. Receptor specificity of the fibroblast growth factor family. Journal of Biological Chemistry 1996271 15292-15297. (https://doi.org/10.1074/jbc.271.25.15292)

22 Jonker JW, Suh JM, Atkins AR, Ahmadian M, Li P, Whyte J, He M, Juguilon H, Yin YQ, Phillips CT, et al. A PPAR $\gamma$-FGF1 axis is required for adaptive adipose remodeling and metabolic homeostasis. Nature 2012485 391-394. (https://doi.org/10.1038/nature10998)

23 Hutley L, Shurety W, Newell F, McGeary R, Pelton N, Grant J, Herington A, Cameron D, Whitehead J \& Prins J. Fibroblast growth factor 1: a key regulator of human adipogenesis. Diabetes $20045 \mathbf{5}$ 3097-3106. (https://doi.org/10.2337/diabetes.53.12.3097)

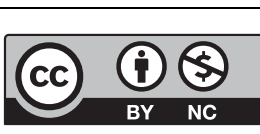

This work is licensed under a Creative Commons Attribution-NonCommercial 4.0 International License. 
24 Scarlett JM, Rojas JM, Matsen ME, Kaiyala KJ, Stefanovski D, Bergman RN, Nguyen HT, Dorfman MD, Lantier L, Wasserman DH, et al. Central injection of fibroblast growth factor 1 induces sustained remission of diabetic hyperglycemia in rodents. Nature Medicine 2016 22 800-806. (https://doi.org/10.1038/nm.4101)
25 Mejhert N, Galitzky J, Pettersson AT, Bambace C, Blomqvist L, Bouloumié A, Frayn KN, Dahlman I, Arner P \& Rydén M. Mapping of the fibroblast growth factors in human white adipose tissue. Journal of Clinical Endocrinology and Metabolism 201095 2451-2457. (https:// doi.org/10.1210/jc.2009-2049)

Received in final form 3 July 2018

Accepted 9 July 2018

Accepted Preprint published online 10 July 2018 http://www.endocrineconnections.org https://doi.org/10.1530/EC-18-0141

(c) 2018 The authors Published by Bioscientifica Ltd

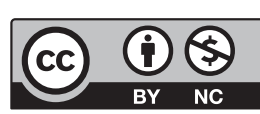

This work is licensed under a Creative Commons Attribution-NonCommercial 4.0 International License. 